\title{
Released power in a vortex-antivortex pairs annihilation process Released power in a vortex-antivortex pairs annihilation process
}

\author{
Cristian Aguirre-Tellez ${ }^{1}$, Miryam Rincón-Joya ${ }^{2 a}$, José José Barba-Ortega ${ }^{2 b}$ \\ ${ }^{1}$ Departamento de Física, Universidad Federal de Mato Grosso, Brasil. \\ Orcid: 0000-0001-8064-6351. Email: cristian@ fisica.ufmt.br \\ ${ }^{2}$ Grupo de Física Mesoscópica, Departamento de Física, Universidad Nacional de Colombia, Colombia. \\ Orcid: a 0000-0002-4209-1698, b 0000-0003-3415-1811. Emails: ${ }^{\mathrm{a}}$ mrinconj@ unal.edu.co, b jjbarbao@unal.edu.co
}

Received: 19 April 2020. Accepted: 23 August 2020. Final version: 5 November 2020.

\begin{abstract}
In this paper, we studied the power dissipation process of a Shubnikov vortex-antivortex pair in a mesoscopic superconducting square sample with a concentric square defect in presence of an oscillatory external magnetic field. The time-dependent Ginzburg-Landau equations and the diffusion equation were numerically solved. The significant result is that the thermal dissipation is associated with a sizeable relaxation of the superconducting electrons, so that the power released in this kind of process might become calculated as a function of the time. Also, we analyzed the effect that the Ginbzurg-Landau $\kappa$ and deformation $\tau$ parameters have on the magnetization, dissipate power and superelectrons density.
\end{abstract}

Keywords: Ginzburg-Landau; mesoscopic; magnetization; vortices.

\section{Resumen}

En este trabajo, estudiamos el proceso de disipación de calor de un par vórtice-antivortice de Shubnikov en un cuadrado superconductor mesoscópico con un defecto cuadrado concéntrico en presencia de un campo magnético externo oscilatorio. Las ecuaciones dependientes del tiempo de Ginzburg-Landau y la ecuación de difusión se resolvieron numéricamente. El resultado principal es que la disipación térmica está asociada con una relajación considerable de los electrones superconductores de modo que la potencia liberada en este tipo de proceso podría calcularse en función del tiempo. Además, analizamos el efecto que tienen los parámetros de Ginzburg-Landau $\kappa$ y deformación $\tau$ dentro del defecto, sobre la magnetización, potencia disipada y densidad de superelectrónes.

Palabras clave: Ginzburg-Landau; mesoscópico; magnetización; vórtices.

\section{Introduction}

The magnetic properties of type $I$ and type $I I$ are given by the Ginzburg - Landau parameter $\kappa$, the superconductor being of type $I I$ when $\kappa>1 / \sqrt{ } 2$ and of type $I$ if $\kappa<1 / \sqrt{ } 2$. Tinkham [1] first showed, that even of material with $\kappa<1 / \sqrt{ } 2$ can behave like type $I$ if the film is sufficiently thin. The manipulation of single, multi, and giant vortex states in mesoscopic superconductors are very important for applications of spins, fluxtronic, medicine, etc $[2,3,4,5,6,7]$. It is possible that a vortex anti vortex pairs becomes stable, this stability correspond to the symmetry of the system with pinning centers $[8,9]$, magnetic dots $[10,11]$, arrays 
of small current loops, hot spot. As is well know, in this kind of process the heat is always present, the heat is diffused and depend on the velocity of such diffusion [12, $13,14]$.

Gulevich and Kusmartsev, proposed a experiment based on a sample with a Josephson coupling term, where the dynamics of vortex and antivortex take place [15]. Sardella et al, analyzed the annihilation of a vortexantivortex pair in a square superconducting system with a central pinning center. Show that when the vortex enters the superconducting sample, its average velocity is $\sim 103 \mathrm{~ms}^{-1}$ and during the annihilation process, its average velocity is $\sim 105 \mathrm{~ms}^{-1}$ [8].

Zadorosny et al studied the dynamics of a vortexantivortex pair in several superconducting systems with different areas and with an antidot inserted in the center, they found that in the annihilation process the vortexantivortex pair acquire an elongated format [9].

E. Duarte et al, studied the vortex antivortex annihilation in a mesoscopic superconducting system with a central pinning center, they found a dissipative effect, generating appreciable changes in the order parameter, and the power released in the annihilation of a vortex-antivortex pair is detectable in measurements of the total magnetic moment as a function of time [16].

J. Barba-Ortega et al, investigated the process of annihilation of a vortex-antivortex pair in a superconducting square with a central anti-pinning center. They found that when a metallic anti-pining center is used the vortex-antivortex collision occurs close to the edge of the defect, and the antivortex speed decrease when the anti-dot becomes more metallic [17].

In this work we studied the dissipative process (total energy released in the collision) between a vortexantivortex collision in a superconducting square with a concentric hole. We calculated the magnetization and density of superconducting electrons as a function of the time for several values of the Ginzburg - Landau parameter. This work is organized as follows. In section 2 we present the theoretical formalism used to study the mesoscopic systems. In section 3 we analyze the results obtained from the simulations and, subsequently, discuss them. In section 4, we present our conclusions.

\section{Theoretical Formalism}

We solve the time dependent Ginzburg Landau equations (TDGL), proposed by Schmid [18], provides a temporal evolution of the order parameter $\psi$ and the potential vector $\mathrm{A}$ for a superconducting sample immersed in an external applied magnetic field $H$. For our purposes, we solved the equations for the energy dissipated due to both the applied magnetic field and the order parameter $(\psi)$ during the vortex-antivortex process $[19,20,21,22,23$, 24]:

$$
\begin{gathered}
\frac{\partial \psi}{\partial t}=-(i \nabla+\mathrm{A})^{2} \psi+\psi\left(\tau(x, y)-|\psi|^{2}\right) \\
\frac{\partial \mathrm{A}}{\partial \mathrm{t}}=\mathrm{J}_{\mathrm{s}}-\kappa^{2}(x, y) \nabla \times \nabla \times A
\end{gathered}
$$

In the equations 1 and $2, \mathrm{~J}_{\mathrm{s}}=\operatorname{Re}[\bar{\psi}(-i \nabla-\mathrm{A}) \psi]$, is the super-current density, $\psi$ represents the order parameter, $A$ the vector potential, $T$ temperature. How is usual we presented, in dimensionless form with $|\psi|$ in units of $\psi_{\infty}=\sqrt{-\frac{\alpha}{\beta}}$ with $\beta=1$, lengths in units of the coherence length $\xi, T=0 ; A$ in units of $H_{c 2} \xi$, where $H_{c 2}$ is the second critical field, temperature in units of the critical temperature $T_{c}$ and time in units of time Ginzburg-Landau tGL $=\pi h / 8 K_{B} T_{c} \eta \cdot \tau(x, y)$ is the deformed parameter $\alpha$ explain the variation of the Cooper pairs density locally in the sample, even this modified model have been added in superconducting samples for different specific geometries. In the Ginzburg-Landau model, $\alpha$ represents regions at different $T_{c}$, and $\kappa(x, y)$ is the Ginzburg-Landau Parameter for an anysotropic sample. We simulated a superconducting square of size $L=12 \xi$ with a central defect with size $L_{d}=2 \xi$, respectively, immersed in an oscillatory magnetic field as (See Figure 1):

$$
H(t)=H_{0} \sin (\omega t)
$$

In the equation $3, H$ goes to $0 \rightarrow 1$, and then the magnetic field is reversed to $1 \rightarrow-1$, and finally the magnetic loop is completed to $-1 \rightarrow 0$. We take $H_{0}=1,0$. The equation for the dissipated power energy was obtained by using the Helmholtz free energy for a superconductor in presence of external magnetic field. Such equation, in dimensionless form, is given by:

$$
\begin{aligned}
W_{\text {total }}=2\left(\frac{\partial A}{\partial t}\right)^{2} & \\
& +\frac{2 u}{{\sqrt{1+\gamma^{2}|\psi|}}^{2}}\left[\left(\left|\frac{\partial \psi}{\partial t}\right|\right)^{2}\right. \\
& \left.+\frac{\gamma^{2}}{4}\left(\left|\frac{\partial|\psi|^{2}}{\partial t}\right|\right)^{2}\right]
\end{aligned}
$$

The first term in the equation 4 represents the dissipation due to the induced electrical field $E$, and the second one is due to the dissipation related to the relaxation of $\psi$. The dissipated power energy is given in units of $H_{c 2}^{2}(0) /\left[8 \pi \kappa^{2}\right.$ 
$\left.t_{G L}\right]$ As $W_{\text {total }}$ diffuses through the system, we couple the thermal diffusion equation to the Ginzburg-Landau ones $[12,16]$. We take $u=5,79$ and $\gamma=10$ (for more details see Ref. [25]). We studied two scenarios with $\kappa=1,0$ and $\tau=1,0$ in the whole sample, except in a) the cases where $\kappa d=0,4,0,7,0,9,1,1$ within the defect, b) the cases where $\tau=0,4,0,7,0,9,1,1$ within the defect. Note that, given the thin film geometry, this is an effective $\kappa$ value, which depends on the thickness of the stripe.

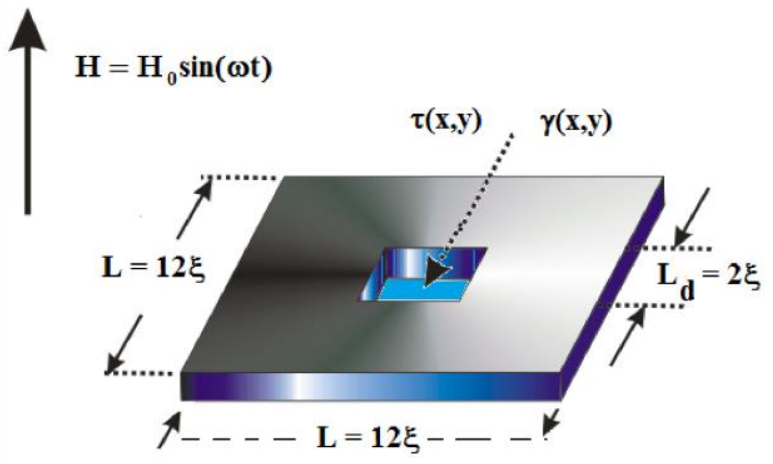

Figure 1. Layout of the studied sample, a superconducting square of lateral size $L=12 \xi$, with a central square defect of size $L_{d}$. The defect is at different temperatures and Ginzburg-Landau parameter. The sample is immersed in a perpendicular oscillatory magnetic field $H=H_{0} \sin (w t)$.

\section{Results and discussion}

In the Figure 2 we show the magnetization loops as a function of the applied magnetic field, $-4 \pi M(H)$, for $\kappa=$ 1,0 in whole sample and a) $\tau=0,4$ b) $\tau=0,7$, c) $\tau=0,9$, and d) $\tau=1,1$. We calculate the vortex number into the sample, we found that, due to the size of the defect and the sample, just one vortex nucleate into the sample at $H \simeq 0,9$ being trapped by the defect, as the magnetic field is decreased, this vortex leaves the sample and when $H$ is inverted, an antivortex enter the sample while a vortex remains trapped in the defect.

Thus, a vortex-antivortex pair is formed and each specimen moves toward each other until their mutual annihilation. A similar process occurs in taking $\tau=0,9$ and $\tau=1,1$, respectively, but in these cases two and six vortices penetrates the sample. As we can see, while $\tau$ increases into the defect, the number of jumps in the magnetization curve increases, and as is well known, the number of jumps in the magnetization curve is related to the number of vortices entering or leaving the sample.

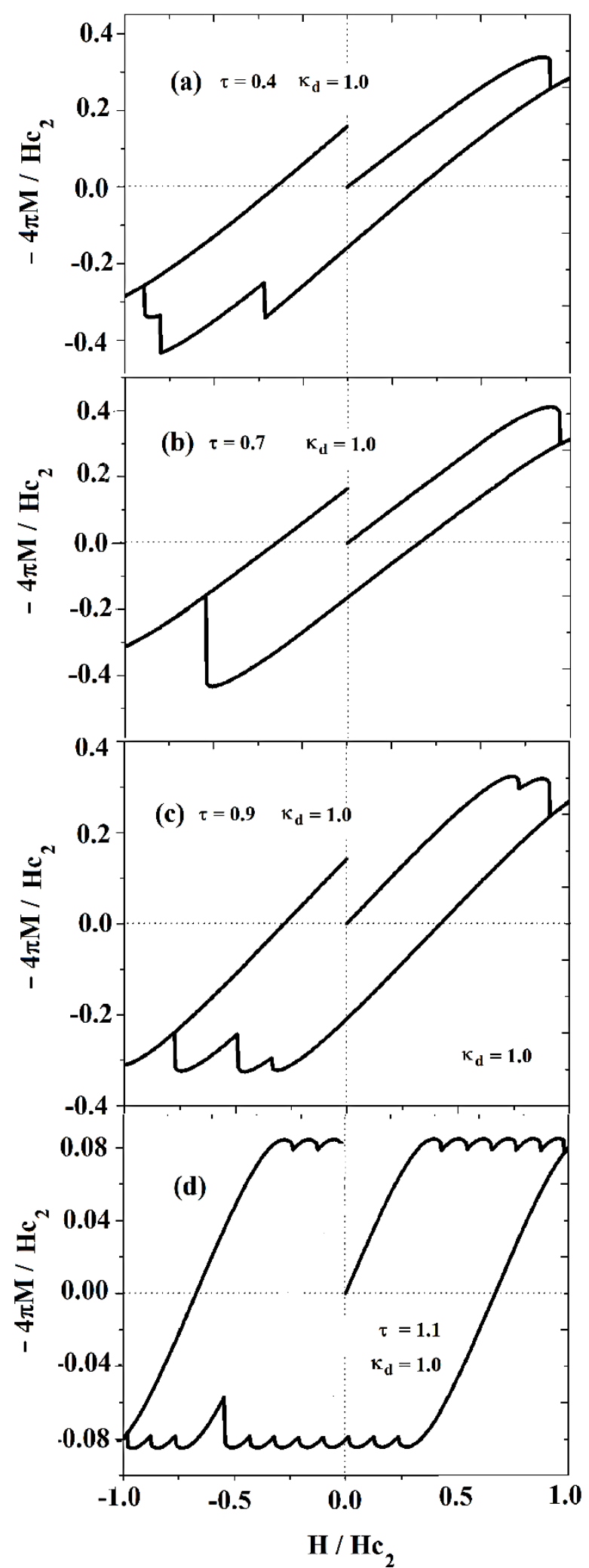

Figure 2. Magnetization loop $-4 \pi M / H_{c 2}$, as a function of the magnetic field $H$, for $\kappa=1,0$ in whole sample and a) $\tau=0,4$ b) $\tau=0,7$, c) $\tau=0,9$, and d) $\tau=1,1$. 
A similar behaviour we can appreciate in the Figure 3, where the loop for the magnetization is plotted for $\tau=1,0$ in whole sample and a) $\kappa d=0,9$, b) $\kappa d=1,1$.

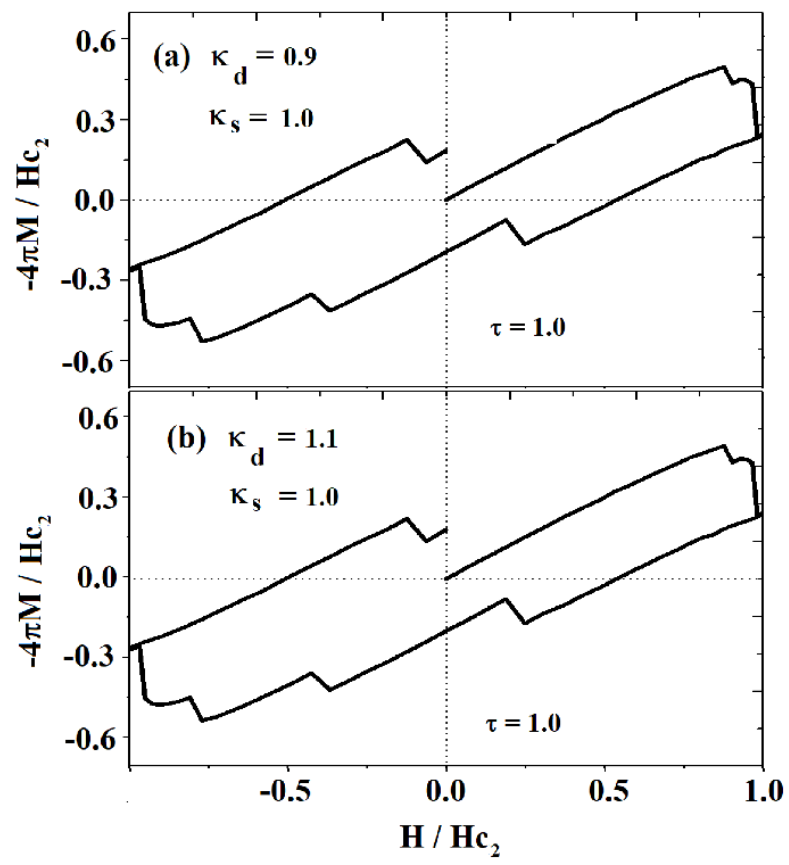

Figure 3. Magnetization loop $-4 \pi M / H_{c 2}$, as a function of the magnetic field $H$, for $\tau=1,0$ in whole sample and a)

$$
\kappa d=0,9, \text { b) } \kappa d=1,1 \text {. }
$$

In this case the effect of $\kappa d$ on the vortex number that penetrates the sample is negligible, a paramagnetic effect in the down branch of the magnetization curve, evidence the pinning vortex effect of the nature of the defect. In the Figure 4 we plot the average of the modulus square of the order parameter $|\psi|^{2}$ as a function of time for $\kappa=$ 1,0 in whole sample and a) $\tau=0,4$ b) $\tau=0,7$, c) $\tau=0,9$, and d) $\tau=1,1$.

As is well know, $|\psi|^{2}$ depends on the vorticity $N$ of the system and presents a sequence of periodic pulses and the number of maximus increases with $\tau$. We calculated the power spectrum $\left(2 W_{\text {Total }}\right)$, Figure 5 , as a function of time $t$, for $\kappa=1,0$ in whole sample and a) $\tau=0,4$ b) $\tau=0,7$, c) $\tau=0,9$, and d) $\tau=1,1$.

For a defect filled with another superconductor at higher Tc $(\tau>1,0)$, the time averaged power spectrum presents an harmonic behaviour as a function of time $t$ for 4 maximus, whereas for a defect filled with a metallic material $0<\tau<1,0$, one and two maximus or the first and second harmonic can be distinguished.
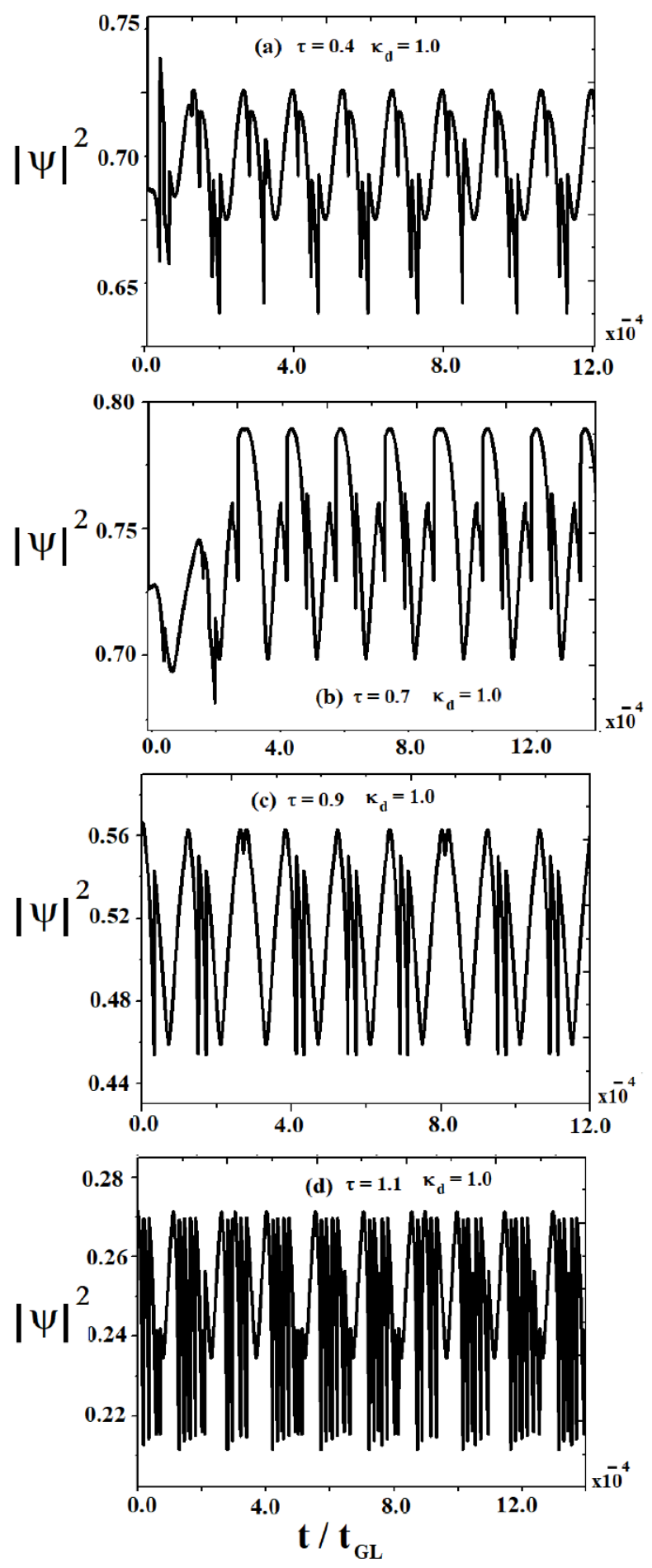

Figure 4. Average of the modulus square of the order parameter $|\psi|^{2}$ as a function of time for $\kappa=1,0$ in whole sample and a) $\tau=0,4$ b) $\tau=0,7$, c) $\tau=0,9$, and d) $\tau=1,1$. 

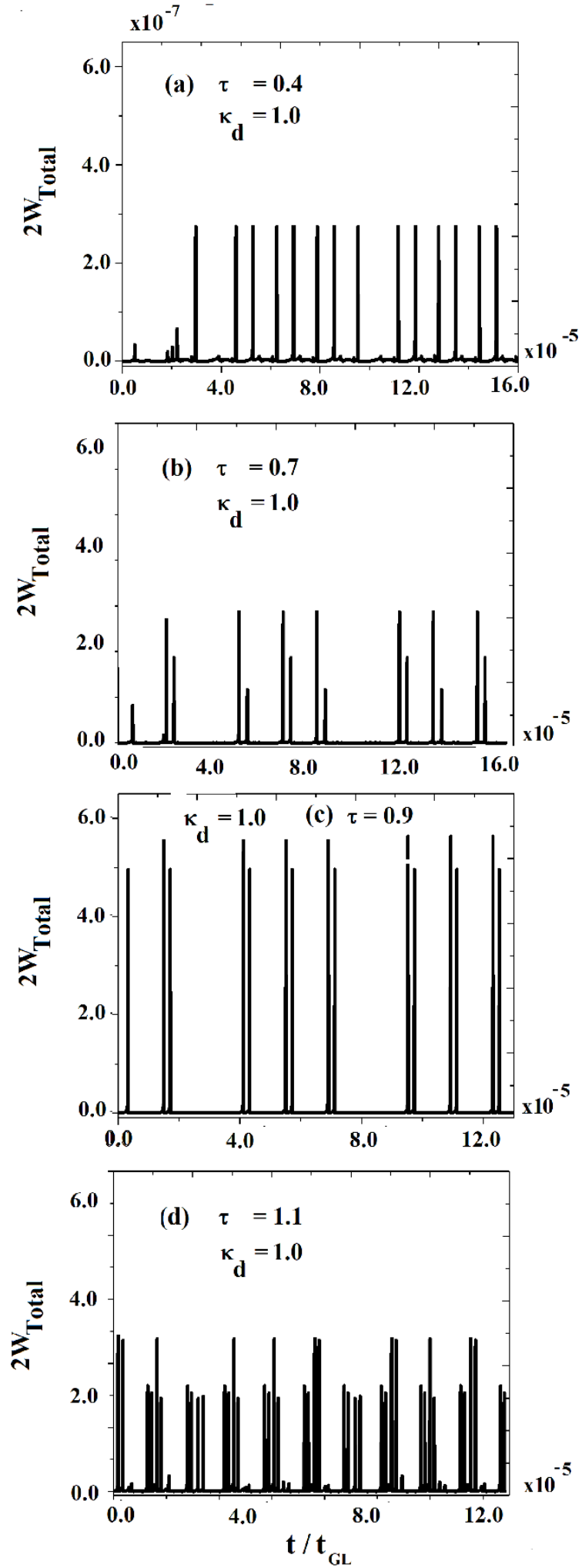

Figure 5. Power $2 W_{\text {total }}$ as a function of time for $\kappa=$ 1,0 in whole sample and a) $\tau=0,4$ b) $\tau=0,7$, c) $\tau=$ 0,9 , and d) $\tau=1,1$.
A similar behaviour is appreciated in the Figure 6 where the average of the modulus square of the order parameter $|\psi|^{2}$ as a function of time for $\tau=1,0$ in whole sample and a) $\kappa_{d}=0,9$, b) $\kappa_{d}=1,1$. A similar behaviour occurs when $\kappa_{d}$ varies into the sample, but the number of maximum in the order parameter independent on $\kappa_{d}<1,0$. We observed an anomalous behaviour in this oscillations when $\kappa_{d}=1,1$ is used. When $\kappa d$ changes into the sample, remains $\tau$ invariant, the power spectrum $\left(2_{\text {WTotal }}\right)$, Figure 7 , as a function of time $t$, is very similar in all te studied cases for $0<\tau<1,0$, and present a non-conventional behaviour for $\tau>1,0$.

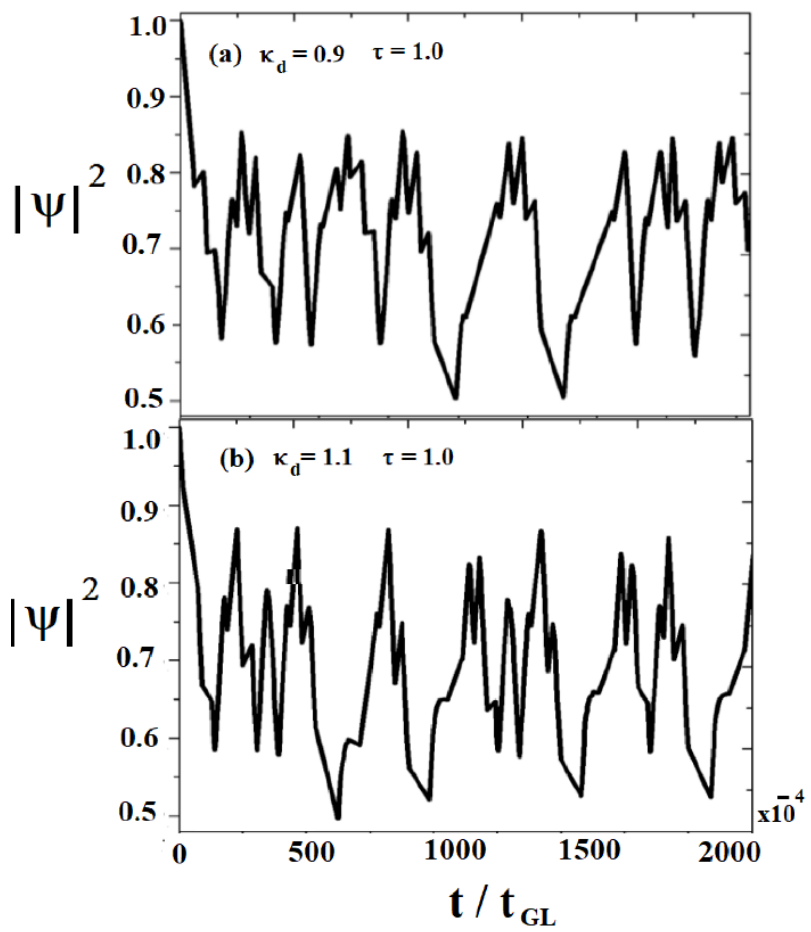

Figure 6. Average of the modulus square of the order parameter $|\psi|^{2}$ as a function of time for $\tau=1,0$ in whole sample and a) $\kappa_{d}=0,9$, b) $\kappa_{d}=1,1$.

\section{Conclusions}

In the thin film limit we studied the released power energy in a vortex-antivortex process in superconducting square with a central defect at several temperatures and Ginzburg-landau parameter. We showed that when the temperature inside the defects increases, the number of maximum in the dissipated power increases with $\tau$, while the Ginzburg-Landau parameter varies into the defects, remains the temperature invariant, the number of maximum is independent of $\kappa_{d}$. We obtained profiles of the average order parameter as a function of time for several $\kappa$ and $\tau$ and calculated their power spectrum. 

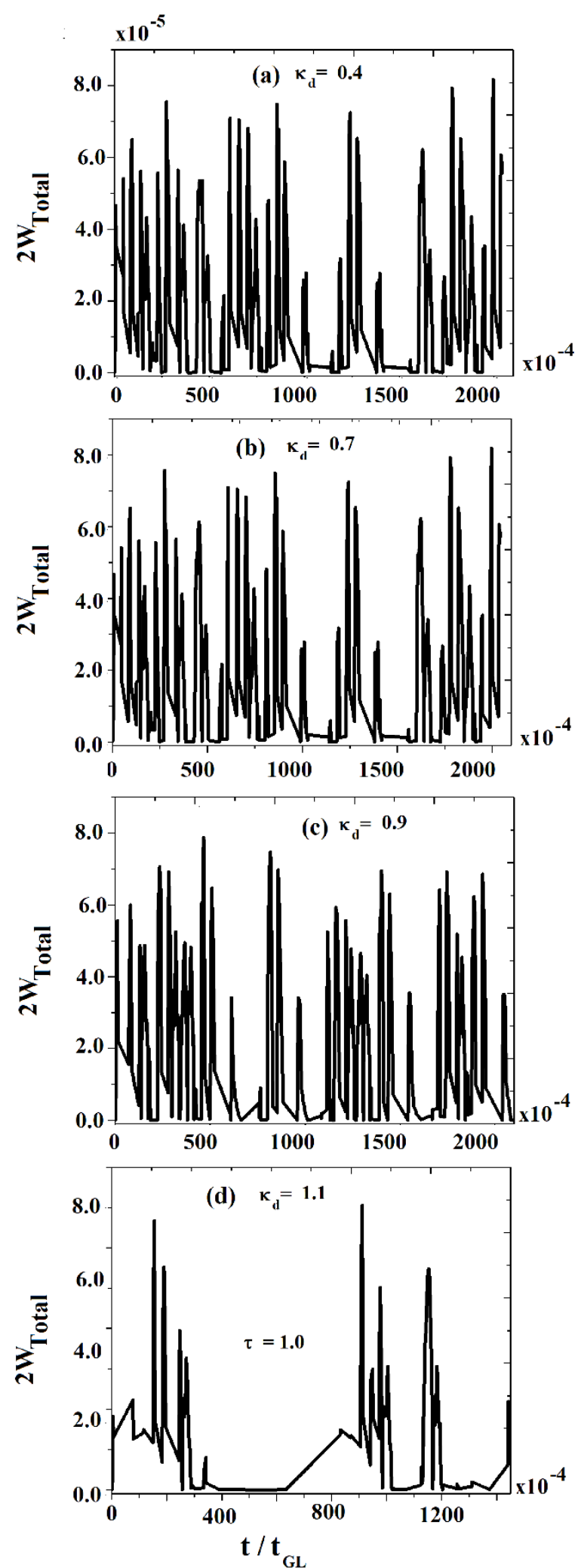

Figure 7. Power $2 W_{\text {total }}$ as a function of time for $\tau=1,0$ in whole sample and a) $\kappa_{d}=0,4$ b) $\kappa_{d}=0,7$, c) $\kappa_{d}=0,9$, and d) $\kappa_{d}=1,1$.
We found that, three harmonic frequency are obtained for $\tau<1,0$, the power spectrum is affected by the entry and exit of vortices and anti-vortices in the defect. Since that the released power in these process are not desirable for several applications of superconducting systems, we claim that the detailed knowledge about vortexantivortex dynamics is a important and relevant element to avoid them. Therefore, our results could contribute to important applications of technological devices.

\section{Acknowledgments}

The Colombian Geological Survey, Laboratory Direction Cali - Colombia, and the Gualconda's gold mine Manager Mrs. Rolberto Álvarez.

\section{References}

[1] M. Tinkham, "Effect of fluxoid quantization on transitions of superconducting films," Physical Review, no. 129, p. 2413, 1963, doi: 10.1103/PhysRev.129.2413

[2] L. Komendova, M. V. Milosević, A. A. Shanenko, F. M. Peeters, "Different length scales for order parameters in two-gap superconductors: Extended Ginzburg-Landau theory," Physical Review B, no. 84, p. 064522, 2011, doi: 10.1103/PhysRev.129.2413

[3] E. D. V. Nino, A. D. Lantada, J. Barba-Ortega, "Vortex matter in a superconducting square under 2D thermal gradient," Journal of Low Temperature Physics, no. 195 , p. 202, 2019, doi: 10.1007/s10909-019-02158-x

[4] C. A. Aguirre, H. Blas, J. Barba-Ortega, "Mesoscale vortex pinning landscapes in a two component superconductor," Physica C, no. 554, p. 8, 2018, doi: 10.1016/j.physc.2018.08.010

[5] G. R. Berdiyorov, X. H. Chao, F. M. Peeters, H. B. Wang, V. V. Moshchalkov, B. Y. Zhu, "Magnetoresistance oscillations in superconducting strips: A Ginzburg-Landau study," Physical Review B, no. $86, \quad$ p. 224504, 2012, doi: 10.1103/PhysRevB.86.224504

[6] G. Berdiyorov, A. R. de C. Romaguera, M. V. Milosević, M. M. Doria, L. Covaci, F. M. Peeters, "Dynamic and static phases of vortices under an applied drive in a superconducting stripe with an array of weak links," Europhysics Journal B, no. 85, p. 130, 2012, doi: 10.1140/epjb/e2012-30013-7

[7] G. R. Berdiyorov, M. V. Milosević, M. L. Latimer, Z. L. Xiao, W. K. Kwok, F. M. Peeters, "Large magnetoresistance oscillations in mesoscopic superconductors due to currentexcited moving vortices," 
Physical Review Letters, no. 109, p. 057004, 2012, doi: 10.1103/PhysRevLett.109.057004

[8] E. Sardella, P. N. L. Filho, C. C. Silva, L. R. E. Cabral, W. A. Ortiz, "Vortex-antivortex annihilation dynamics in a square mesoscopic superconducting cylinder," Physical Review B, vol. 80, p. 012506, 2009, doi: 10.1103/PhysRevB.80.012506

[9] R. Zadorosny, E. C. S. Duarte, E. Sardella, W. A. Ortiz, "Vortex-antivortex annihilation in mesoscopic superconductors with a central pinning center," Physica $C$, vol. 503, p. 94, 2014, doi: 10.1016/j.physc.2014.04.007

[10] A. Gomes, E. M. Gonzalez, D. A. Gilbert, M. V. Milosević, L. Kai, J. L. Vicent, "Probing the dynamic response of antivortex, interstitial and trapped vortex lattices on magnetic periodic pinning potentials," Superconductor Science and Technology, no. 26, p. 085018, 2013, doi: 10.1088/0953-2048/26/8/085018

[11] A. V. Kapra, V. R. Misko, D. Y. Vodolazov, F. M. Peeters, "The guidance of vortex antivortex pairs by inplane magnetic dipoles in a superconducting finite size film," Superconductor Science and Technology, no. 24, p. 024014, 2011, doi: 10.1088/0953-2048/24/2/024014

[12] V. N. Gladilin, J. Tempere, J. T. Devreese, V. V. Moshchalkov, "Stable antiferromagnetic vortex lattice imprinted into a type-II superconductor," New Journal of Physics, no. 14, p. 103021, 2012,

[13] I. Shapiro, E. Pechenik, B. Y. Shapiro, "Recovery of superconductivity in a quenched mesoscopic domain," Physical Review B, vol. 63, p. 184520, 2001, doi: 10.1103/PhysRevB.63.184520

[14] M. Ghinovker, B. Y. Shapiro, I. Shapiro, "Spontaneous magnetic-flux generation in superconducting ring," Physical Review B, vol. 53, p. 240, 2001, doi: 10.1209/epl/i2001-00143-x

[15] D. R. Gulevich, F. V. Kusmartsev, "Fluxon collider for multiple fluxon antifluxon collisions," New Journal of Physics, no. 9, p. 59, 2007, doi: 10.1088/13672630/9/3/059

[16] E. C. S. Duarte, E. Sardella, W. A. Ortiz, R. Zadorosny, "Dynamics and heat diffusion of abrikosovs vortex antivortex pairs during an annihilation process," Journal of Physics: Condensed Matter, no. 29, p. 40, 2017.
[17] J. Barba-Ortega, E. Sardella, J. A. Aguiar, "Vortex antivortex dynamics in a mesoscopic superconducting prism with a centered antidot," Journal of Superconductivity and Novel Magnetism, no. 24, p. 97, 2011, doi: 10.1007/s10948-010-0904-8

[18] A. Schimd, "A time dependent Ginzburg - Landau equation and its application to the problem of resistivity in the mixed state," Physik der Kondensierten Materie, no. 5, p. 302, 1966, doi: 10.1007/BF02422669

[19] I. Petkovic, A. Lollo, L. I. Glazman, J. G. E. Harris, "Deterministic phase slips in mesoscopic superconducting rings," Nature Communicatios, no. 7, p. 13551, 2016, doi: 10.1038/ncomms 13551

[20] C. A. Aguirre, Q. Martins, J. Barba-Ortega, "Desarrollo analítico de las ecuaciones Ginzburg Landau para películas delgadas superconductoras en presencia de corrientes," Revista UIS Ingenierías, no. 18, p. 213, 2019, doi: 10.18273/revuin.v18n2-2019020

[21] J. Barba-Ortega, E. Sardella, J. A. Aguiar, "Superconducting properties of a parallelepiped mesoscopic superconductor: A comparative study between the 2D and 3D ginzburglandau models," Physics Letters A, no. 379, p. 732, 2015, doi: 10.1016/j.physleta.2014.12.030

[22] C. A. Aguirre, M. R. Joya, J. Barba-Ortega, "Dimer structure as topological pinning center in a superconducting sample," Revista UIS Ingenierías, no. 19, p. 119, 2020, doi: 10.18273/revuin.v19n1-2020011

[23] W. D. Gropp, H. G. Kaper, G. K. Leaf, D. M. Levine, M. Palumbo, V. M. Vinokur, "Numerical simulation of vortex dynamics in type-II superconductors," J. Comput. Phys., vol. 123, p. 254, 1996, doi: 10.1006/jcph.1996.0022

[24] G. C. Buscaglia, C. Bolech, A. López, Connectivity and Superconductivity. Springer, Berlin, 2000.

[25] L. Kramer, R. J. Watts-Tobin, "Theory of dissipative current-carrying states in superconducting filaments," Phisical Review Letteres, no. 40, p. 1041, 1978, doi: 10.1103/PhysRevLett.40.1041 